\title{
EXPERIMENTAL OBSERVATION OF A 100-FEMTOSECOND SINGLE ELECTRON BUNCH IN PHOTOCATHODE LINAC WITH LONGITUDINAL EMITTANCE COMPENSATION TECHNIQUE
}

\author{
J. Yang", K. Kan, T. Kondoh, T. Kozawa, Y. Yoshida, S. Tagawa The Institute of Scientific and \\ Industrial Research, Osaka University, \\ 8-1 Mihogaoka, Ibaraki, Osaka 567-0047, Japan
}

\begin{abstract}
The realization of a $100 \mathrm{fs}$ electron pulse is important for the studies of ultrafast physical/chemical phenoena with a pump-probe method. We have developed a photocathode linear accelerator (linac) to generate such electron pulse with a magnetic bunch compressor. The nonlinear effect of the magnetic fields in the pulse compression was compensated carefully by optimizing the magnetic fields and the booster linac RF phase. A 98 fs electron bunch in rms with electron charge of $0.17 \mathrm{nC}$ was observed experimentally by using a femtosecond streak camera. The beam energy was $32 \mathrm{MeV}$, and the normalized teraservers emittance was lower than $3.8 \mathrm{~mm}$ mrad. The dependences of the bunch length, the relative energy spread and the emittance on the electron charge were also measured.
\end{abstract}

\section{INTRODUCTION}

High-brightness electron sources, producing short, intense, low-emittance electron bunches, are key elements for new developments in accelerator physics, such as future high-energy electron-positron colliders, laser or plasma wakefield acceleration, and new femtosecond $\mathrm{x}$ ray free electron lasers (FELs) based on self-amplified spontaneous emission (SASE). The x-ray FEL project at DESY and the LCLS at SLAC will use the electron bunches compressed down to $80 \mathrm{fs}$, and the SPPS will use bunches as short as $30 \mathrm{fs}$ [1].

Ultrashort electron bunches, of the order of $100 \mathrm{fs}$, are also essential to reveal the hidden dynamics of intricate molecular and atomic processes through experimentation such as time-resolved electron diffraction and femtochemistry. Most of the electron-induced ultrafast reactions or phenomena are investigated by a timeresolved pump-probe technique. In the pump-probe experiments, a short electron bunch is used as a pump source. The electron-induced reactions are analyzed generally with a synchronized ultrashort probe light such as femtosecond lasers or $\mathrm{x}$-rays [2]. A femtosecond single electron bunch with beam energy of a few tens $\mathrm{MeV}$ is very important to be utilized in this technique for observing information of the most basic reaction mechanisms in physics, chemistry and biology (e.g. excitation, ionization, and relaxation of atoms and molecules) on the femtosecond time scale.

In this paper, we report the experimental observation of femtosecond single electron bunching in a photoinjector with a magnetic compressor. In the photoinjector, a photocathode radio frequency (rf) gun was used to produce a picosecond electron bunch with low transverse emittance. A booster linear accelerator (linac) after the gun was used to accelerate the bunch up to about $32 \mathrm{MeV}$ with an optimal energy-phase correlation in the bunch for the best compression in magnetic field. Finally, the electron bunch was compressed into femtosecond by transporting it through the magnetic compressor, which rotates the bunch in the longitudinal phase space distribution.

\section{EXPERIMENTAL ARRANGEMENT}

\section{Photocathode RF Gun and Laser}

Figure 1 shows the femtosecond electron bunch generation system. A 1.6-cell S-band (2856MHz) RF gun, produced by Sumitomo Heavy Industries (SHI) $[3,4]$ as the Gun IV type at Brookhaven National Laboratory (BNL), was used in the system. The RF gun was composed of two cells: a half cell and a full cell. The length of the half cell was designed to be 0.6 times the full cell length to reduce the beam divergence. The coupling between the waveguide and cavity was located in the full cell. Coupling between the cells was accomplished via the iris of the cavity. The copper cathode used in the system was located on the side of the half cell. A single solenoid magnet was mounted at the exit of the RF gun to compensate the space charge emittance. The cathode magnetic field was measured to be less than $10 \mathrm{G}$ at a peak magnetic field of $3 \mathrm{kG}$, resulting in a negligible emittance growth due to the cathode magnetic field.

The RF gun was driven by an all solid-state LDpumped Nd:YLF picosecond laser. The oscillator was mode-locked with a frequency of $79.3 \mathrm{MHz}$, the $36^{\text {th }}$ subharmonic of the $2856 \mathrm{MHz}$ accelerating RF, by adjusting the cavity length of the oscillator with a semiconductor saturable absorber mirror (SESAM). The time jitter between the oscillator output and the reference $79.3 \mathrm{MHz}$ $\mathrm{RF}$ signal was measured to be $<0.5$ ps using a phase detector technique. The ultraviolet (UV) light, which was frequency quadrupled to a $262 \mathrm{~nm}$ using a pair of nonlinear crystals with the maximum energy of $0.3 \mathrm{~mJ}$, was injected on the cathode surface at an incident angle of approximately $2^{\circ}$ along the direction of the electron beam using a prism placed downstream of the gun, as shown in Fig.1. The diameter of the beam size at the cathode

\#yang@sanken.osaka-u.ac.jp 
surface was $2 \mathrm{~mm}$. The pulse width of the UV light was measured to be 5 ps (FWHM) by a streak camera.

\section{Linear Accelerator and RF source}

The electron beam produced by the RF gun was accelerated with a $2 \mathrm{~m}$ long S-band travelling-wave linear accelerator (linac). The linac was located at a distance of $1.2 \mathrm{~m}$ from the cathode surface. The energy of the electron pulse was also modulated by adjusted the RF phase for pulse compression, as described below. The operating temperatures of the RF gun and the linac were $32^{\circ} \mathrm{C}$ and $30^{\circ} \mathrm{C}$, respectively. The temperature fluctuation of both the RF gun and the linac were within $0.1^{\circ} \mathrm{C}$.

The peak RF inputs of the RF gun and the linac were 10 MW and $25 \mathrm{MW}$, respectively, which was produced by a 35 MW Klystron. The stability of the RF power was $0.1 \%$ peak-to-peak. The effective pulse width of the RF was 4 $\square \mathrm{s}$. The peak on axis electric fields in the RF gun and the linac were approximately 115 and $20 \mathrm{MV} / \mathrm{m}$, respectively. The repetition rate of the operation was $10 \mathrm{~Hz}$ in the experiment. A high-power phase shifter installed in a 25 MW RF line, as shown in Fig. 1, was used to adjust the $\mathrm{RF}$ phase of the linac for energy modulation.

\section{Magnetic Bunch Compressor}

The magnetic bunch compressor, as shown in Fig. 1, was constructed with two $45^{\circ}$-bending magnets and four quadrupole magnets (two pairs), which provides the necessary path length dependence on energy. The picosecond electron bunch, which was produced in the linac with an energy-phase correlation, was compressed into femtosecond by rotating the bunch in the longitudinal phase space distribution. In order to obtain a short bunch length, all magnets were carefully installed with the minimum lattice error to reduce the aberrations in the phase space distribution. The inside two quadrupole magnets had equal magnetic fields, while the outside quadrupole magnets had equal magnetic fields. The dispersion function is symmetric on the midplane of the compressor. However, during the compression, higherorder momentum dispersion, especially the second-order dispersion, causes a nonlinear deformation of the longitudinal phase space, which increases the final bunch length. To reduce the nonlinear effects, we used the curvature of the $\mathrm{rf}$ waveform in the linac. By re-phasing the linac away from the zero-crossing of the rf (i.e. away from the linear slope), a nonlinear energy correlation along the bunch length can be introduced. The correlation can offset the effects of the nonlinear path length in the magnetic compression by optimizing the magnetic fields of four quadrupole magnets.

\section{Bunch Length Measurement}

The compressed bunch length was obtained by measuring Cherenkov radiation emitted from the electron bunch in air at the exit of the compressor with a femtosecond streak camera (HAMAMATSU, FESCA200, C6138). The optical measurement system to guide the Cherenkov radiation to the streak camera, as shown in Fig. 1, consisted of a thin Al mirror and two convex lenses with focal length of $20 \mathrm{~cm}$. Its optical pass length was about $2 \mathrm{~m}$. An optical band pass filter (BPF), which is centered at $480 \mathrm{~nm}$ and has a band width of $11 \mathrm{~nm}$ in FWHM, was used to reduce the radiation pulse broadening due to optical dispersion. A $15 \mu \mathrm{m}$-wide slit in the streak camera was used in the measurement to avoid the pulse broadening due to space charge effect in the camera. The time resolution of the streak camera in the measurement time region of 20 ps was $183 \mathrm{fs}$ in FWHM and 78 fs in rms.

\section{RESULTS AND DISCUSSIONS}

Figure 2 gives a temporal distribution of the electron bunch measured by the streak camera at charge of 0.17 nC. The rms bunch length was obtained to be 98 fs by fitting the bunch shape to a Gaussian distribution. In the measurement, the linac was re-phased at $94^{\circ}$ off crest of the rf waveform, which was the optimum phase for the shortest bunch length. The rf phase of the gun was fixed at $30^{\circ}$ off zero-crossing rf phase to produce a lowemittance beam.

Figure 3 gives a plot of the rms compressed bunch length versus the bunch charge. The bunch lengths were

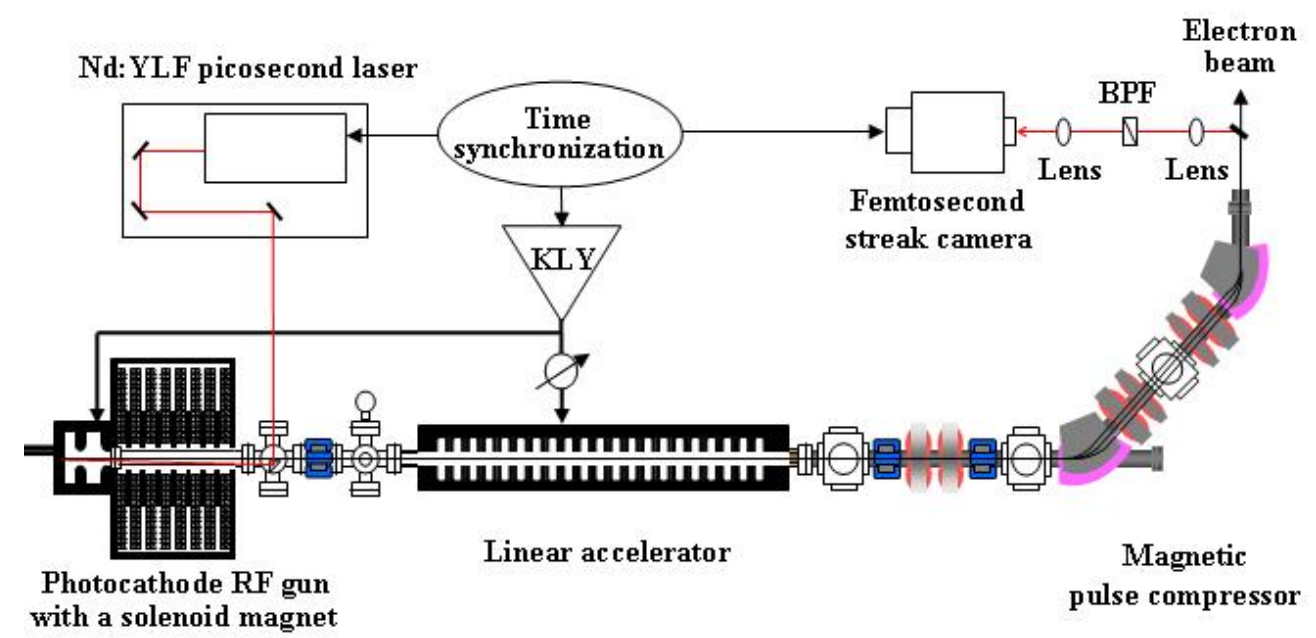

Figure 1: Schematic diagram of the experimental arrangement for the generation of femtosecond electron bunch. 
measured under the constant linac phase of $94^{\circ}$, but the four quadrupole magnetic fields were optimized to obtain the minimum bunch length at each charge. We found that the bunch length increase, which is independent on the bunch charge, is negligible in the compression. The bunch length increase due to the space charge effect is very close to the analytical expression in ref. [5], $\mathrm{Q}^{2 / 3}$, where $\mathrm{Q}$ is the bunch charge. However, the final compressed bunch length is also determined with the longitudinal emittance of the initial beam [6]. The space charge effect in the rf gun causes the longitudinal emittance growth, resulting in the bunch length increase in Fig. 2.

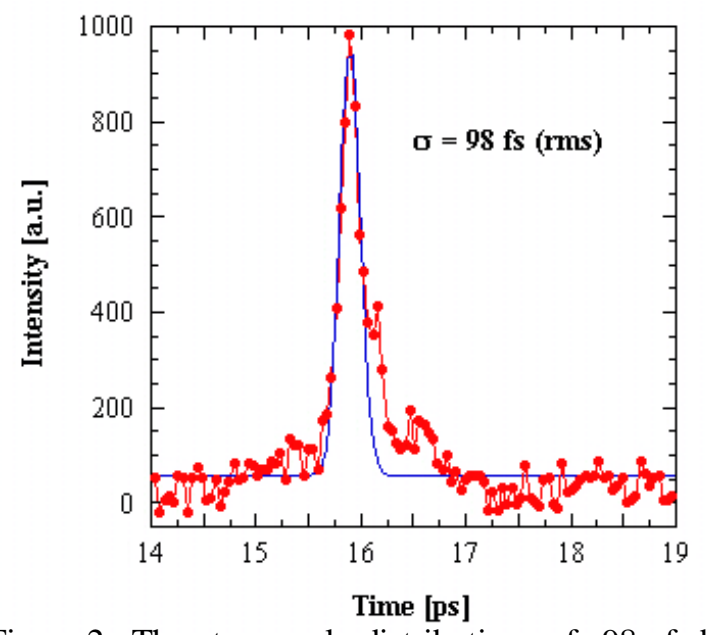

Figure 2: The temporal distribution of 98 fs-bunch measured by the streak camera at $0.17 \mathrm{nC}$.

The energy spread of the beam downstream of the linac were measured by using the first $45^{\circ}$ bending magnet and a $30 \mu$ m-thick screen which was made of sintered $\mathrm{Al}_{2} \mathrm{O}_{3}$ doped with $\mathrm{Cr}$ and mounted on the midplane of the compressor. It was found that the energy spread of the beam is slightly dependent on the bunch charge. The relative energy spread was increased from $0.2 \%$ to $0.35 \%$ when we increased the bunch charge from $0.17 \mathrm{nC}$ to 1.25 $\mathrm{nC}$. The beam energy was $32 \mathrm{MeV}$ at the exit of the linac.

The transverse emittance was measured downstream of the linac and upstream of the bunch compressor with a

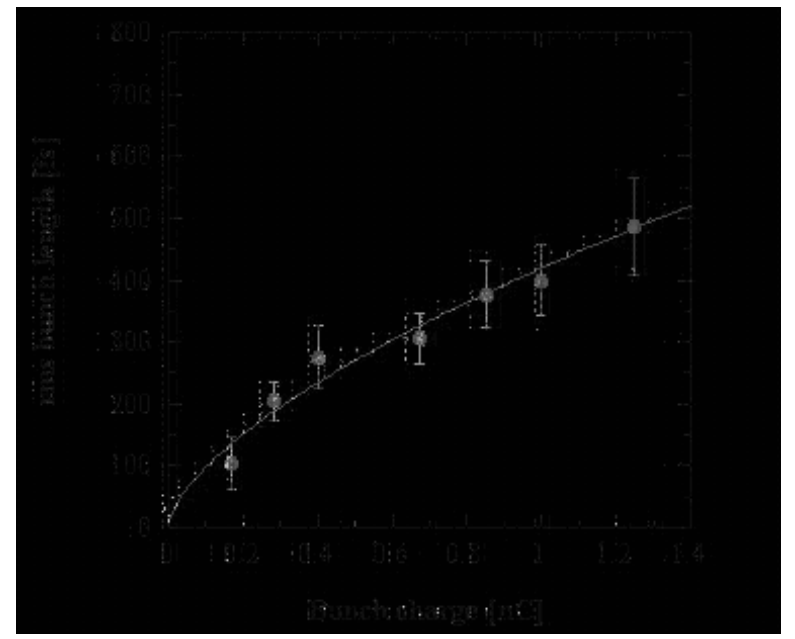

Figure 3: The dependence of the rms bunch length on the bunch charge. standard quadrupole scan technique. Figure 5 gives the plot of the normalized rms transverse emittance versus the bunch charge under a constant solenoid magnetic field of $1.71 \mathrm{kG}$ which produced the lowest emittance at the bunch charge of $1.0 \mathrm{nC}$. The rf phase in the rf gun was fixed to $30^{\circ}$ and $94^{\circ}$ in the linac. A linear dependence of the transverse emittance on the bunch charge was observed. The emittance due to space charge effect was increased linearly with the bunch charge at a rate of 1.2 $\mathrm{mm}-\mathrm{mrad} / \mathrm{nC}$.

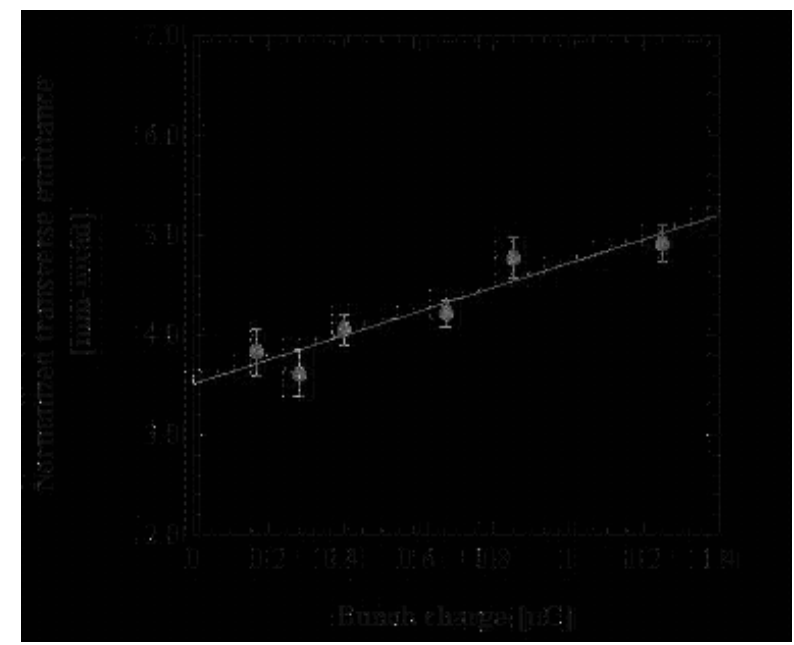

Figure 4: The normalized rms transverse emittance under a constant solenoid magnetic field of $1.71 \mathrm{kG}$.

\section{CONCLUSIONS}

In summary, the presented work demonstrates the generation of ultrashort single electron bunch in a photoinjector with a magnetic compressor by rotating the bunch in the longitudinal phase space distribution. A nonlinear energy correlation along the bunch length produced in booster linac was successfully used to compensate the nonlinear effects in the magnetic fields. An electron bunch with an rms bunch length of 98 fs was achieved for a $32 \mathrm{MeV}$ electron beam with the relative energy spread of $0.2 \%$ and the normalized transverse emittance of 3.8 $\mathrm{mm}$-mrad at electron charge of $0.17 \mathrm{nC}$. The effects of space charge on the bunch length, the relative energy spread and the normalized beam transverse emittance were investigated experimentally.

\section{REFERENCES}

[1] L. Bentson, et al., Nucl. Instrum. Methods Phys. Res. Sect. A 507, 205 (2003).

[2] Y. Yoshida, et al., Radit. Phys. Chem., 60 (2001), 313-318.

[3] J. Yang, et al., J. Appl. Phys., 92, 1608-1612 (2002).

[4] J. Yang, et al., Nucl. Instrum. Meth. Phys. Res. Sect. A 491, 15-22 (2002).

[5] J.B. Rosenzweig, N. Barov and E. Colby, IEEE Trans. Plasma Sci. 24, 409 (1996).

[6] K. J. Kim, Nucl. Instrum. Methods Phys. Res. Sect. A 275, 201 (1989). 